\title{
The feasibility of ultrasound-guided vacuum- assisted evacuation of large breast hematomas
}

\author{
Sa’ed Almasarweh¹, Mazen Sudah¹, Sarianna Joukainen², Hidemi Okuma1, \\ Ritva Vanninen ${ }^{1,3}$, Amro Masarwah ${ }^{1}$ \\ ${ }^{1}$ Kuopio University Hospital, Diagnostic Imaging Center, Department of Clinical Radiology, Kuopio, Finland \\ ${ }^{2}$ Kuopio University Hospital, Department of Plastic Surgery, Division Surgery, Kuopio, Finland \\ 3 University of Eastern Finland, Cancer Center of Eastern Finland, Kuopio, Finland
}

Radiol Oncol 2020; 54(3): 311-316.

Received 3 May 2020

Accepted 27 May 2020

Correspondence to: Sa'ed Almasarweh, Department of Clinical Radiology, Kuopio University Hospital, Puijonlaaksontie 2, P.O. Box 100, FI-70029 Kuopio, Finland. E-mail: saadn.masarweh@gmail.com

Disclosure: No potential conflicts of interest were disclosed.

Background. Breast hematoma is an often underrated and disregarded post-procedural complication in the literature. Current treatment modalities are comprised of either surgical or expectant therapy, while percutaneous procedures play a smaller role in their treatment. We aimed to examine the efficacy of vacuum-assisted evacuation (VAE) in the treatment of clinically significant large breast hematomas as an alternative to surgery.

Patients and methods. We retrospectively analysed patients that underwent breast interventions (surgical and percutaneous), who later developed clinically significant large hematomas and underwent a trial of VAE of hematoma in our hospital within the period of four years. Patient and procedure characteristics were acquired before and after VAE. Success of intervention was based on $\geq 50 \%$ clearance of hematoma volume and patients' subjective resolution of symptoms. All patients were followed clinically and by ultrasound if needed at different intervals depending on the severity of presenting symptoms.

Results. Eleven patients were included in the study. The mean largest diameter of hematomas was $7.9 \mathrm{~cm}$ and mean surface area was $32.4 \mathrm{~cm}^{2}$. The mean duration of the procedure was $40.5 \mathrm{~min}$. In all patients VAE of hematoma was implemented successfully with no complications. Control visits showed no major residual hematoma or seroma formation.

Conclusions. Our results show that VAE of hematoma can be implemented as a safe alternative to surgery in large, clinically significant hematomas, regardless of aetiology or duration. The procedure carries less risk, stress and cost with the added benefit of outpatient treatment when compared to surgical treatment.

Key words: breast hematoma; vacuum assisted breast biopsy; hematoma evacuation; breast

\section{Introduction}

Complications following therapeutic, reconstructive, or aesthetic breast surgeries as well as percutaneous procedures, both biopsies and excisions, are important considerations for women undergoing or pursuing these options. In general, the most common local complications following routine breast interventions are inherent to the surgery itself $e . g$. infection, pain, hematoma, delayed heal- ing, and abnormal scarring. Risk factors for complications include smoking, obesity, larger breasts, anticoagulant treatments, and older age. ${ }^{1}$

Early clinically significant postoperative hematomas typically develop within the first 12 to 48 hours after surgery. ${ }^{2}$ Immediate reoperation is usually indicated in expanding hematomas, hemodynamic instability, and jeopardized flap viability. ${ }^{3}$ Less commonly, a hematoma appears days or weeks after surgery and may be associated 
with minor injury or trauma to the breast, with the majority identified within the first 14 days. Late hematomas can also occur and are thought to be related to direct trauma, clotting disorders, overactivity, and use of intraoperative corticosteroids. ${ }^{4,5}$ Postprocedural hematomas are not uncommon, yet most of them are small and resolve spontaneously. Large, clinically more significant hematomas in the late postoperative period are infrequent. Symptomatic, painful, or infected hematomas are treated surgically since hematomas with dense contents or clots do not drain with needle aspirations or drains are blocked immediately. ${ }^{6}$

If a clinically significant hematoma does occur, an evacuation is advised. Expectant management is not favoured due to the lengthy nature of spontaneous liquefaction and discomfort that patients report, which eventually leads to fibrosis and distortion of breast tissue. ${ }^{7}$ Surgical management is aimed at the rapid decompression of the closed wound space through exploration, drainage and establishing haemostasis. After evacuation the wound is thoroughly irrigated and closed in order to preserve the cosmetic aspect of the breast. ${ }^{8}$ Percutaneous drainage of the hematoma during the first 24 hours of hematoma formation might be challenging, on the assumptions that an organized clot would have already been formed. Partial liquefaction occurs 6 to 7 days after the formation of the hematoma, which is considered as the best interval for percutaneous evacuation. ${ }^{9}$

Breast imaging-guided interventions are widely used in daily practice e.g. core biopsy and vacuum assisted breast biopsy and excision (VABB and $\mathrm{VABE}$ ) to diagnose different types of imaging findings and remove benign or risk lesions. The larger the needle used for biopsy and the number of cores obtained, the more likely complications will appear. ${ }^{10}$ Significant vascular damage is more probable in VABB or VABE procedures. ${ }^{11}$ Recently it was reported that VABB can be used as a treatment modality for clinically significant hematoma in patients with small hematomas less than $4 \mathrm{~cm}$ in size. ${ }^{6}$ The effectiveness of vacuum-assisted evacuation of large breast hematomas has not been previously reported.

In this study we aimed to investigate ultrasound-guided vacuum-assisted evacuation (VAE) of breast hematoma as a safe, viable, time and resource-sparing treatment modality for larger (> $5 \mathrm{~cm}$ ) breast hematomas irrespective of aetiology. This technique could decrease the rate of multiple operations and eliminate added morbidity of surgery and anaesthesia while yielding satisfactory therapeutic results.

\section{Patients and methods}

\section{Patients}

All VAE of hematomas performed in our institution between February 2016 and February 2020 were retrospectively retrieved from the regional picture archiving and communication system (PACS) and the clinical data of these patients were also retrieved from the local digital archives. In our institution, hematomas that do not fulfil the criteria for immediate surgery, cause discomfort and unsettling symptoms for the patients (considerable pain, pressure symptoms, local infection and prolonged healing) or patients who refuse surgical intervention are offered a trial of VAE of hematoma. The Chair of the Hospital District waived the need for written informed consent from the patients due to the retrospective nature of this study. All clinical investigations were conducted according to the relevant guidelines and the principles expressed in the Declaration of Helsinki.

\section{Data collection}

The total amount of breast surgeries, VABB and $\mathrm{VABE}$ as well as postoperative haemorrhagic complications requiring surgical intervention were retrieved from the hospital's digital information systems.

All medical records of patient undergoing VAE procedures were also reviewed and the following parameters were recorded and included in a database: Age, type of breast procedure, time interval between previous procedure and VAE of hematoma, symptoms exhibited pre- as well as post-VAE, medications, comorbidities, size of hematoma and the estimated residual volume after the procedure, echogenicity of hematoma at time of VAE, gauge of the needle used, complications during or after the procedure and findings at control. The total duration of the procedure was measured from the time the patient has entered the ultrasound room until discharge.

\section{Procedure}

In Kuopio University Hospital (KUH), automated VABB procedures were introduced in 2015. With experience in VABB, VABE was gradually introduced and consequently evacuation of large hematomas was offered as an alternative to surgery. The procedures were carried out with EnCor ${ }^{\mathrm{TM}}$ Breast Biopsy System (BD Bard, Tempe, AZ, USA). US-guided interventions were performed using 


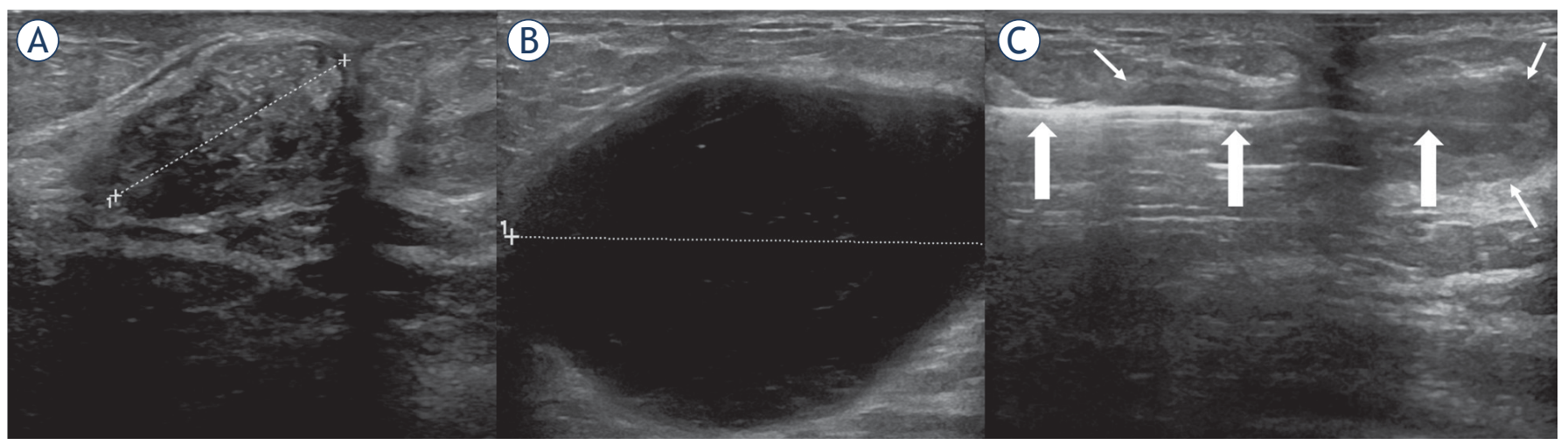

FIGURE 1. Illustration of a vacuum assisted evacuation of hematoma in patient number 9 . Vacuum assisted excision of a discordant lesion (A) at core biopsy resulted in a palpable painful hematoma. Ultrasound image of the $5.5 \times 4.0 \mathrm{~cm}$ hematoma (B). Complete evacuation of the hematoma with sparing of hematoma wall (C); Large arrows indicate needle's shaft and small white arrows indicate hematoma wall

a Logiq E9 class US scanner (GE, Wauwatosa, Wisconsin, USA) equipped with a $5-15 \mathrm{MHz}$ linear array transducer. All procedures were performed by, or under the supervision of, a breast radiologist with over 25 years of experience in multimodality breast imaging and interventions. No change in patients' medications was required. After thorough local disinfection, application of aseptic measures as well as the injection of local anaesthetic through the insertion channel (lidocaine with adrenaline; max $10 \mathrm{ml}$ ) a small skin incision was made. An EnCor ${ }^{\mathrm{TM}} 7 / 10 \mathrm{G}$ vacuum needle was then inserted into the base of the hematoma. The needle's cutting blade was opened, and continuous suction was applied until the hematoma emptied and its walls collapsed (Figure 1). Residual hematomas in side-pockets were ignored. In the case of incomplete hematoma aspiration (less than 50\%) due to large, blocking or hard clots, the blade was used to fragment the fibrotic tissue through multiple biopsy samples. Sample container was continuously flushed with saline during the aspiration-fragmentation procedure to avoid blockage, and the container would be changed if filled with accumulated material as needed. The walls of the hematoma were carefully avoided during any fragmentation procedure to avoid possible rebleeding. After the procedure, the area of the breast with hematoma was manually compressed for at least 10 minutes, longer, if the patient received anti-coagulants. The use of a tight sports-brassiere was recommended for a minimum of 24 hours with compression pads over the area of the hematoma to prevent rebleeding or major seroma formation. Since 2019 we continued to provide fully adjustable and flexible breast compression wraps to all patients. Patients were then discharged.
Some hematomas were longer than the shaft of the vacuum needle, thus separate insertions from opposite sides were implemented to complete the procedure. Otherwise, procedures were completed through single insertion.

Success of intervention was based on clearance of a targeted $\geq 50 \%$ of hematoma's volume, visually estimated by the operator, patients' subjective assessment of symptom resolution and the resolution of hematoma without the need for surgery during follow-up. All patients were followed clinically and by US if needed at different intervals depending on the severity of presenting symptoms.

\section{Results}

During the recruitment period, a total of 1208 breast operations and $358 \mathrm{VABB}$ or lesion excision procedures were performed. We detected a total of 44 clinically large hematomas as complications. Of the 1208 operative patients, 33 had early postoperative bleeding and had to undergo surgical evacuation while 8 patients suffered from delayed hematoma formation. Therefore, the reoperation rate for early postoperative bleeding was $2.7 \%$ $(33 / 1208)$ and the rate of late hematomas treated with VBE was $0.7 \%(8 / 1208)$. On the other hand, of the 358 patients that have undergone VABB and excision procedures, 3 patients were later diagnosed to have clinically relevant hematomas with an incidence rate of $0.84 \%(3 / 358)$.

Altogether 11 consecutive patients who have been diagnosed with breast hematoma and treated with the VAE system were included in the analysis. Patients had a mean age of 59 years (range 38-85) and their characteristics are presented in Table 1. 
TABLE 1. Characteristics of patients with hematoma

\begin{tabular}{cccccc}
\hline Patient & $\begin{array}{c}\text { Age } \\
\text { (yrs) }\end{array}$ & $\begin{array}{c}\text { Wait time* } \\
\text { (days) }\end{array}$ & Intervention & Medications & $\begin{array}{c}\text { Size } \\
(\mathbf{c m})\end{array}$ \\
\hline 1 & 42 & 14 & BLES & Anti-Coagulant & $6 \times 4$ \\
2 & 67 & 1 & VABB & None & $8 \times 4$ \\
3 & 38 & 36 & Surgery & None & $6 \times 3$ \\
4 & 48 & 78 & Surgery & None & $6 \times 5$ \\
5 & 49 & 21 & Surgery & None & $5.5 \times 3$ \\
6 & 51 & 34 & Surgery & $\begin{array}{c}\text { Anti-Platelet and } \\
\text { hydrocortisone }\end{array}$ & $7 \times 6.5$ \\
7 & 84 & 15 & Surgery & Anti-Platelet & $12 \times 2.5$ \\
8 & 71 & 597 & Surgery & None & $5.5 \times 3.5$ \\
9 & 85 & 51 & VABE & Anti-Coagulant & $5.5 \times 4$ \\
10 & 60 & 29 & Surgery & None & $20 \times 5$ \\
11 & 53 & 22 & Surgery & None & $5.5 \times 5$ \\
\hline
\end{tabular}

$*$ Wait time $=$ number of days between surgical intervention/biopsy and VAE of hematoma;

BLES $=$ breast lesion excision system; $\mathrm{VABB}=$ vacuum assisted breast biopsy; VABE $=$ vacuum assisted breast-lesion excision

TABLE 2. Hematoma characteristics pre- and post-vacuum assisted evacuation (VAE)

\begin{tabular}{cccl}
\hline Patient & $\begin{array}{c}\text { Size pre-VAE } \\
\text { (in cm) }\end{array}$ & $\begin{array}{c}\text { Estimated decrease in size } \\
\text { post-VAE (percentage) }\end{array}$ & Symptoms \\
\hline 1 & $6 \times 4$ & $>50 \%$ & Resolution \\
2 & $8 \times 4$ & $>50 \%$ & Resolution \\
3 & $6 \times 3$ & $100 \%$ & Resolution \\
4 & $6 \times 5$ & $100 \%$ & Resolution \\
5 & $5.5 \times 3$ & $100 \%$ & Resolution \\
6 & $7 \times 6.5$ & $80 \%$ & Resolution \\
7 & $12 \times 2.5$ & $70 \%$ & Resolution \\
8 & $5.5 \times 3.5$ & $90 \%$ & Resolution \\
9 & $5.5 \times 4$ & $100 \%$ & Resolution \\
10 & $20 \times 5$ & $80 \%$ & Resolution \\
11 & $5.5 \times 5$ & $100 \%$ & Resolution \\
\hline
\end{tabular}

Of the 11 participants, 3 patients had hematomas as complications after percutaneous interventional procedures and 8 patients after surgeries, of which 5 were reduction mammoplasties. The mean number of days between the initial intervention and VAE of hematoma of 10 patients was 30 days with an outlier of 597 days due to an idiopathic late developing complicated hematoma in a mastectomy site after radiotherapy. One of these patients had a slowly progressive hematoma after VABB and refused surgical evacuation. Regarding symptoms prior to evacuation, all patients reported pain,
$45.5 \%$ prolonged healing $(n=5), 45.5 \%$ mass effect $(\mathrm{n}=5), 18.2 \%$ infection $(\mathrm{n}=2)$.

Of the ultrasound imaging of the hematomas taken prior to VAE of hematoma, 7/11 were hypoechoic, $1 / 11$ was hyperechoic and $3 / 11$ had mixed echogenicity. All hematomas underwent unsuccessful aspiration trials with fine needles (G18-23). The mean duration of the VAE procedure was 40.5 min (range 19-62). One patient was taking aspirin alone, one aspirin and hydrocortisone, one patient was taking Warfarin, one patient taking Dabigatran and one patient taking Apixaban.

One patient had a massive two-sided communicating $20 \times 5$ and $12 \times 4 \mathrm{~cm}$ hematoma, therefore in this analysis, we included only the largest portion. The mean maximum diameter of the evacuated hematomas was $7.9 \mathrm{~cm}$ and an average surface area of $32.4 \mathrm{~cm}^{2}$. The gauge of the VAE probes was a choice between 7G or 10G. Most of the procedures were performed using $7 \mathrm{G}$-sized needles $(\mathrm{n}=8)$ owing to the larger size of the treated hematomas. Four patients underwent ultrasound-guided aspiration of the hematoma cavity due to post-evacuation seroma formation 1-7 days after VAE procedure. No complications were reported post-evacuation or aspiration procedures.

The parameters before as well as after VAE of hematoma are depicted in Table 2. All patients underwent regular follow-up after evacuation. Upon follow-up, all cases were deemed successfully treated with no major hematoma residue or seroma formation.

\section{Discussion}

One of the most common complications in breast interventions is hematoma formation, which remains grossly underrated and disregarded in the literature, especially when its frequency is taken into consideration. Breast hematomas can range from small mammographically-detected hematomas to large clinically significant hematomas that can cause severe discomfort to patients. Our results suggest that Vacuum-assisted evacuation of hematoma is a time-sparing, cost-effective and successful method of evacuation for small as well as large breast hematomas regardless of aetiology.

Our patient population was comprised of both post-biopsy and post-surgical patients, thus expanding the aetiological factors to not only include biopsy-induced hematomas. In our study we included all consecutive patients treated in our institution presenting with hematomas of different 


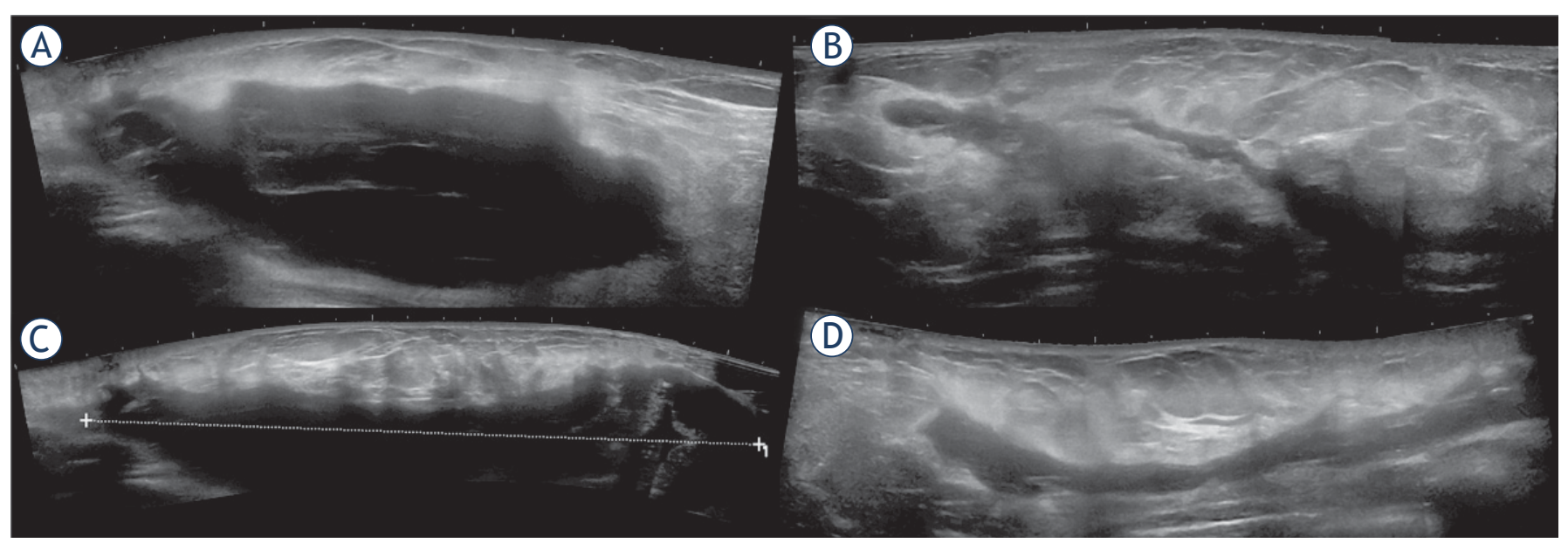

FIGURE 2. Patient number 10 with massive two-sided communicating hematoma treated through separate punctures. Image (A) represents a panoramic view of the cranial aspect of the $12 \mathrm{~cm}$ long hematoma and image (B) represents a panoramic view after the evacuation. Image (C) represents the caudal $20 \mathrm{~cm}$ long hematoma and correspondingly image (D) shows the view after treatment.

sizes. This goes to prove that VAE can be implemented in clinically large significant hematomas. It is stipulated that percutaneous drainage of acute hematomas should be attempted between days 7-14 after formation of hematoma, in order to allow time for hematoma liquefaction. Moreover, delayed hematomas, i.e. hematomas developing 6 months or more post-intervention, are always evacuated surgically.7 In our study the wait time was variable, in that the procedure timing was not set on a set-point schedule, rather on different time-intervals regarding date of hematoma formation. Furthermore, one large delayed hematoma was evacuated successfully after 597 days (1.64 years), proving that even delayed hematomas can be successfully treated with VAE of hematoma irrespective of duration.

While the true incidence of large clinically significant hematomas remains unknown, findings from this study show that it is relatively uncommon. The treatment of breast hematomas in the literature is suggested as either surgical or expectant. Both treatment modalities impose certain risks and added morbidity for the patient. Patients face problems such as added costs, the ordeal of going through surgery or stress due to the aesthetic and psychological impact of the procedure. Moreover, expectant therapies may pose future diagnostic difficulties. ${ }^{9}$

A recent report evaluated the efficacy of the VABB system in evacuating symptomatic hematomas after VABB excision of benign breast lesions in 8 patients. Evacuation was successful in all the cases and no technique-related complications were observed. ${ }^{6}$ However, the inclusion criteria were restricted to hematomas observed post-VABB or VABE and not post-surgical complications, which limits the patient population on which this technique can be used. Moreover, 75\% (6/8) of hematomas were smaller than $4 \mathrm{~cm}$ with a largest maximum diameter of $5.6 \mathrm{~cm}$. This means that most clinically significant breast hematomas that are difficult to handle conservatively were excluded. The study failed to address whether this technique could be attempted on larger and more difficult to evacuate hematomas, which would otherwise need to be evacuated surgically.

Vacuum-assisted biopsies are also currently implemented as a treatment modality for small palpable or non-palpable benign or risk lesions, by assuring rapid and complete excision of these lesions to be better histopathologically evaluated and therefore obviating the need for therapeutic surgery or continuous follow-up. ${ }^{12}$ Minimally invasive management of many B3 lesions with VABE continues to be a suitable alternative to first-line surgical excision in most cases. ${ }^{13}$ In this study, we wanted to study the efficacy of automatic VABE system in removing symptomatic clinically significant hematomas. Not only are VABE systems faster with less implications on patients, but the endogenous vacuum capability, the large bore size as well as the slicing mechanism of the probe could be used to evacuate organized hematomas, which otherwise would be difficult to aspirate percutaneously and would need surgical drainage and evacuation.

Evacuations were performed immediately upon request and without prior scheduling as our 
patients presented with acute symptoms and the procedure offered immediate relief. Furthermore, most of these patients were discharged immediately after the procedure. No change in anticoagulant medications were required as the procedures were quite straightforward without any excision of fibroglandular breast tissues. The tip of the blade is very sharp and penetrates even denser tissues easily, hence special care should be applied in handling the needle inside the hematoma cavity in order not to induce any damage to the cavity walls. Due to the large size of the needles, we regularly chose the shortest insertion pathway and used a combination of local anaesthetic with adrenaline to reduce any possible bleeding consequences.

The obvious limitations of the study are the small number of patients and the retrospective nature. This study needs to be validated on a larger scale to include more patients with a more controlled inclusion and outcome criteria. Furthermore, the volume of the evacuated part of the hematoma was not measured during the procedure due to the continuous saline flush used in our practice and could not be retrospectively accurately verified.

To conclude, this study shows that VAE procedure is a successful, time-conserving, easily implemented interventional treatment modality for both small and large breast hematomas that would decrease the morbidity, costs and inconvenience of repeated surgery.

\section{References}

1. Araco A, Gravante G, Araco F, Delogu D, Cervelli V, Walgenbach K. A retrospective analysis of 3,000 primary aesthetic breast augmentations: postoperative complications and associated factors. Aesthetic Plast Surg 2007; 31 532-9. doi: 10.1007/s00266-007-0162-8

2. Seth AK, Hirsch EM, Kim JY, Dumanian GA, Mustoe TA, Galiano RD, et al. Hematoma after mastectomy with immediate reconstruction: an analysis of risk factors in 883 patients. Ann Plast Surg 2013; 71: 20-3. doi: 10.1097/ SAP.0b013e318243355

3. Phan R, Rozen WM, Chowdhry M, Fitzgerald O'Connor E, Hunter-Smith DJ, Ramakrishnan VV. Risk factors and timing of postoperative hematomas following microvascular breast reconstruction: A prospective cohort study. Microsurgery 2020; 40: 99-103. doi: 10.1002/micr.30473

4. Collins JB, Verheyden $\mathrm{CN}$. Incidence of breast hematoma after placement of breast prostheses. Plast Reconstr Surg 2012; 129: 413e-20e. doi: 10.1097/ PRS.0b013e3182402ce0

5. Handel N, Cordray T, Gutierrez J, Jensen JA. A long-term study of outcomes, complications, and patient satisfaction with breast implants. Plast Reconstr Surg 2006; 117: 757-67; discussion 768-72. doi: 10.1097/01. prs.0000201457.00772.1d

6. Guzman-Aroca F, Berna-Serna JD, Garcia-Ortega AA, Hernandez-Gomez D, Berna-Mestre JD. A new management technique for symptomatic haematomas following therapeutic vacuum-assisted biopsy. J Clin Med 2019; 8: 1493. doi: $10.3390 / \mathrm{jcm} 8091493$

7. Smith B. Complications of Breast Surgery. In: Cance WG, editor. Breast surgery Amsterdam: IOS Press; 2001. p. 95-102.
8. Vitug AF, Newman LA. Emergencies in breast surgery. Surg Clin North Am 2007; 87: 431-51. doi: 10.1016/j.suc.2007.01.005

9. Polverini A, Kruper L. Surgical Emergencies in Breast Surgery. In: Fong Y editor. Surgical Emergencies in the Cancer Patient. New York: Springer International Publishing; 2017. p. 431-51.

10. Zagouri F, Gounaris A, Liakou P, Chrysikos D, Flessas I, Bletsa G, et al. Vacuum-assisted breast biopsy: more cores, more hematomas? In Vivo 2011; 25: 703-5.

11. Bick U, Trimboli RM, Athanasiou A, Balleyguier C, Baltzer PAT, Bernathova $\mathrm{M}$, et al. Image-guided breast biopsy and localisation: recommendations for information to women and referring physicians by the European Society of Breast Imaging. Insights Imaging 2020; 11: 12. doi: 10.1186/s13244-0190803-x

12. Park HL, Hong J. Vacuum-assisted breast biopsy for breast cancer. Gland Surg 2014; 3: 120-7. doi: 10.3978/j.issn.2227-684X.2014.02.03

13. Rageth CJ, O'Flynn EAM, Pinker K, Kubik-Huch RA, Mundinger A, Decker T, et al. Second International Consensus Conference on lesions of uncertain malignant potential in the breast (B3 lesions). Breast Cancer Res Treat 2019; 174: 279-96. doi: 10.1007/s10549-018-05071-1. 